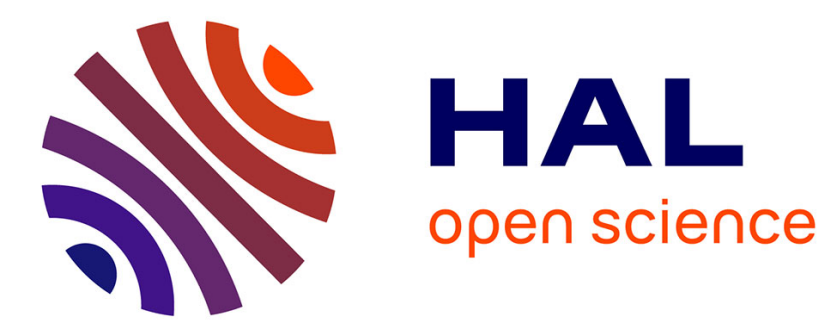

\title{
TDMA-aware Routing Protocol for Multi-hop Communications in Vehicular Ad Hoc Networks
}

Mohamed Hadded, Paul Mühlethaler, Anis Laouiti, Leila Azouz Saidane

\section{To cite this version:}

Mohamed Hadded, Paul Mühlethaler, Anis Laouiti, Leila Azouz Saidane. TDMA-aware Routing Protocol for Multi-hop Communications in Vehicular Ad Hoc Networks. WCNC 2017 - IEEE Wireless Communications and Networking Conference, Mar 2017, San Francisco, United States. hal-01441264

\author{
HAL Id: hal-01441264 \\ https://hal.science/hal-01441264
}

Submitted on 19 Jan 2017

HAL is a multi-disciplinary open access archive for the deposit and dissemination of scientific research documents, whether they are published or not. The documents may come from teaching and research institutions in France or abroad, or from public or private research centers.
L'archive ouverte pluridisciplinaire HAL, est destinée au dépôt et à la diffusion de documents scientifiques de niveau recherche, publiés ou non, émanant des établissements d'enseignement et de recherche français ou étrangers, des laboratoires publics ou privés. 


\title{
TDMA-aware Routing Protocol for Multi-hop Communications in Vehicular Ad Hoc Networks
}

\author{
Mohamed Hadded*†‡, Paul Muhlethaler ${ }^{\dagger}$, Anis Laouiti* and Leila Azouz Saidane ${ }^{\ddagger}$ \\ *SAMOVAR, Télécom SudParis, CNRS, Université Paris-Saclay, 9 rue Charles Fourier 91011 EVRY, France \\ ${ }^{\dagger}$ INRIA, BP 105. 78153 Le Chesnay Cedex, Paris-Rocquencourt, France \\ ${ }^{\ddagger}$ RAMSIS Team, CRISTAL Laboratory, 2010 Campus University, Manouba, Tunisia \\ \{mohamed.hadded, anis.laouiti\}@telecom-sudparis.eu,paul.muhlethaler@inria.fr, leila.saidane@ensi.rnu.tn
}

\begin{abstract}
Vehicular Ad-Hoc Networks (VANETs) have become an emerging technology due to the variety of their applications in Intelligent Transportation Systems (ITS). By creating a vehicular network, each vehicle can exchange information to inform drivers in other vehicles about the current status of the traffic flow or a dangerous situation. Multi-hop communications is an effective method that can be used for information exchange over distances greater than the transmission range of the transmitting vehicle. However, it is a great challenge to ensure a stable multi-hop communication link with a low delivery delay due to the high mobility of the vehicles involved. The goal of this paper is to design a TDMA aware Routing Protocol for Multi-hop wireless vehicular ad hoc networks (TRPM) in order to provide the ability to transmit/receive packets over long distances. The proposed routing scheme is based on a medium access control protocol, in which the intermediate vehicles are selected based on the TDMA scheduling. The simulation results reveal that our routing protocol significantly outperforms other protocols in terms of average end-to-end delay, average number of relay vehicles and the average delivery ratio.
\end{abstract}

Keywords-VANETs, MAC, TDMA, CSMA, multi-hop communications, cross-layer.

\section{INTRODUCTION AND MOTIVATION}

VANETs are deployed to make communication between vehicles possible using ad hoc devices. Nowadays, these networks have become an emerging technology due to the variety of their applications in Intelligent Transportation System(ITS). By crating a vehicular network, each vehicle can exchange information to inform drivers in other vehicles about the current status of the traffic follow or the existence of a dangerous situation [1]. They can also be used to improve traffic management conditions such as route optimization, flow congestion control and to provide on-board infotainment such as Internet access. Since safety applications in VANETs have stringent QoS requirements, an efficient Medium Access Control (MAC) protocol that can provide a broadcast service with bounded access delays and minimum transmission collision is required. Recently, MAC protocols, notably those that are based on the TDMA technique, have been used to enable multiple vehicles to use the same frequency channel without interfering with other vehicles' transmissions [2]. Moreover, a VANET is characterized by its lack of a central coordinator, and thus a data or safety message may pass through multiple intermediate vehicles during its transmission from the source vehicle to the destination vehicle. However, the nodes in VANETs are characterized by their high mobility, so the network topology can change quickly and frequently [1]. These conditions create further difficulty to building and maintaining a multi-hop routing path between the source and destination. Generally, the routing protocols which are proposed for VANETs are designed to find the best path for endto-end packet delivery, which can satisfy QoS requirements by considering the number of relay nodes and link lifetime. Although these protocols can achieve good performance in terms of the metrics studied, they are not simultaneously optimized to maximize the overall network performance [3].

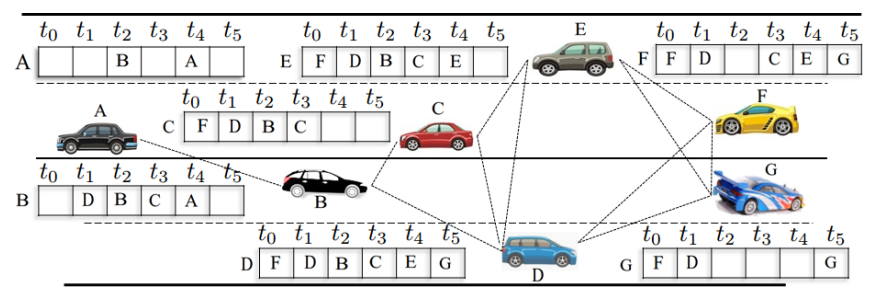

Fig. 1. VANET network using random TDMA scheduling scheme.

In Figure 1, we show an example of a situation where unsuitable routing decisions lead to a large end-to-end delay. The presented VANET scenario consists of 7 vehicles identified by letters (A to $G$ ), using a random TDMA scheduling [4] represented by vectors of length equal to 6 . Each element of a vector represents one time slot that can be used by only one vehicle to send messages. The shortest path in terms of the number of hops provided by the routing protocol does not always ensure the shortest end-to-end delay. For example, when considering vehicle $G$ as the destination vehicle that will broadcast a message collected from vehicle A, the path A-B-D-G is the shortest, in terms of the number of hops, but it produces a delay of 16 time slots (4 time slots to reach slot $t 4$ which is the transmission slot for vehicle $\mathrm{A}$, then 4 time slots between $t 4$ and $t 2$ as $t 2$ is the transmission slot for vehicle $\mathrm{B}$, then 5 time slots between $t 4$ and $t 1$ as $t 1$ is the transmission slot for vehicle D and finally 3 time slots between $t 1$ and $t 5$ as $t 5$ is the transmission slot in which vehicle $G$ will broadcast the message received from vehicle A). This delay is greater than the delay of the path A-B-C-E-G which uses 3 relay nodes and requires 11 time slots (4 time slots to reach slot $t 4,4$ time slots between $t 4$ and $t 2$, then 1 slot between $t 2$ and $t 3,1$ slot between $t 3$ and $t 4$ and 1 slot between $t 4$ and $t 5$ ). In this paper, we present a new TDMA-aware Routing Protocol for Multihop communications, called TRPM that can ensure coherent decisions between the MAC and routing layers by selecting the next relay node based on the DTMAC scheduling scheme [2]. The proposed cross-layer is evaluated in a highway 
scenario by studying the end-to-end delay required to deliver a packet from a source to destination.

The rest of the paper is organized as follows. In Section 2, we review related work. Section 3 describes the system models and briefly presents the DTMAC protocol. Section 4 describes our routing protocol, called TRPM for TDMAaware Routing Protocol for Multi-hop communications in VANETs. In this section, we describe how the message is delivered between a source vehicle and a destination vehicle through multiple relay nodes. Section 5 presents the simulation results and the performance evaluation. Finally, conclusions and future work are reported in Section 6.

\section{RELATED WORK}

The simultaneous transmissions in VANETs due to multiple concurrent vehicles, lead to an increase in the collisions rate which can degrade the network performance in terms of packet delivery ratio and delay. The relevance of this issue has been confirmed by the development of a specific IEEE standard to support VANETs. The IEEE 802.11p [5] which is the emerging standard deployed to enable vehicular communication, is a contention-based MAC protocol, using a priority based access scheme that employs both Enhanced Distributed Channel Access (EDCA) and Carrier Sense Multiple Access with Collision Avoidance (CSMA/CA) mechanisms [6]. This standard is a contentionbased MAC method that cannot ensure a reliable broadcast mechanism with bounded access delays. This disadvantage is particularly detrimental in VANETs which are specially designed to improve road safety. Despite that, several multihop routing protocols use this approach to transmit data. For instance, in [7] the authors have proposed an opportunistic broadcast protocol named OB-VAN to overcome the problem of packet delivery in VANETs. OB-VAN uses a modified 802.11 MAC layer using an active signaling mechanism to select a suitable next-hop relay from all the candidate vehicles that have correctly received the packet. Since OBVAN uses a contention- free MAC schemes, it is possible that it cannot operate well in sparse or dense mobility scenarios.

Many alternatives exist to mitigate collision between vehicle transmissions by using a contention-free protocols at the medium access layer. For instance, the authors in [8] have proposed a cross-layer MAC and routing scheme based on contention-free MAC protocol called VeMAC [9] for multihop in-vehicle Internet access. The goal of this work is to propose a routing protocol which allows a vehicle to discover the existence of a gateway connected to the Internet and exchange packets with it through multi-hop communications. The proposed routing protocol consists of two main phases: gateway discovery and packet forwarding. The first phase defines how a vehicle can discover the existence of a gateway installed along the highway, while the second one defines how a packet is transmitted via multi-hop communications from a vehicle to a gateway and vice versa. However, this multi-hop communication scheme is limited only to infotainment applications (i.e. Internet access) and does not support safety applications, which makes it unsuitable for VANETs which are specially designed to improve road safety. In this paper, we focus on this category of approach and we propose a new TDMA-aware Routing protocol for Multihop Communications in VANETs, in which the next hop decisions are based on the TDMA scheduling at the MAC layer. Unlike [8], our protocol allows a vehicle to send packets over a large distance using V2V communication.

\section{SyStem Model AND DTMAC PROTOCOL}

In this paper, we suppose that each vehicle in a VANET is equipped with a GPS (Global Positioning System) that can allow it to obtain an accurate real-time three-dimensional geographic position (latitude, longitude and altitude), speed and exact time. Moreover, synchronization between vehicles may be performed by using GPS timing information. Each road is divided into $N$ small fixed areas, denoted by $x_{i}, i=$ $1, \ldots, N$ (see Figure 2). Note that the area size depends on the transmission range of the vehicles. We assume that the vehicles are equipped with digital maps to determine which area they are in. Moreover, the vehicles employ multi-hop communications to send their packets over a large distance. As shown in Figure 2, a source vehicle denoted by A, transmits its messages to a destination vehicle denoted by $\mathrm{G}$ through some relay vehicles. In the following, we will briefly describe the TDMA scheduling principle of DTMAC protocol [2]. The latter uses the vehicle location and slot reuse concept to ensure that vehicles in adjacent areas have collision-free schedules. The channel time is partitioned into frames and each frame is further partitioned into three sets of time slots $S_{0}, S_{1}$, and $S_{2}$ of size equal to $n_{0}, n_{1}$ and $n_{2}$, respectively. These sets are associated with vehicles moving in the areas $x_{i}, x_{i+1}$, and $x_{i+2}$, respectively. Moreover, the three subsets of time slots are reused between neighboring areas in such a way that no vehicles in different adjacent areas can access the channel at the same time, and thus no interference can occur. When an active vehicle $v$ moving within the area $x_{i}$ needs to acquire a time slot on the transmission channel, it starts listening to the channel during the set of time slots reserved for the area in which it is traveling, let $S_{j}(v)$, where $j=(i+2) \bmod 3$. At the end of the frame the vehicle $v$ can determine the set of its neighbors $N(v)$ and the set of busy slots in $S_{j}(v)$, denoted by $B(v)$. In order to avoid any collision problem, this set of time slots can not be used by any neighboring vehicles ${ }^{1}$. Therefore, vehicle $v$ can determine the set of available time slots $F(v)$ and then attempt to select one of them at random.

\section{CONTRIBUTION : TDMA-AWARE ROUTING FOR MULTI-HOP COMMUNICATIONS IN VANETS}

In this paper we present a new TDMA-aware Routing Protocol for Multi-hop communications in VANETs, named TRPM. The main idea of this protocol is to select the next hop using the vehicle position and the time slot information from the TDMA scheduling. Like the GPSR protocol [10], we assume that each transmitting vehicle knows the position of the packet's destination. In TRPM, the TDMA scheduling information and the position of a packet's destination are sufficient to make correct forwarding decisions at each transmitting vehicle. Specifically, if a source vehicle is moving in area $x_{i}$, the locally optimal choice of next hop is the neighbor geographically located in area $x_{i+1}$ or $x_{i-1}$ according to the position of the packet's destination. As a result, the TDMA slot scheduling obtained by DTMAC can be used

\footnotetext{
${ }^{1}$ The set of neighbors is the set of vehicles that are moving within the same area.
} 
to determine the set of next hops that are geographically closest to the destination. In fact, each vehicle that is moving in the area $x_{i}$ can know the locally optimal set of next hops that are located in adjacent areas $x_{i+1}$ or $x_{i-1}$ by observing the set of time slots $S_{(i+3) \% 3}$ or $S_{(i+1) \% 3}$, respectively. We consider the same example presented above when vehicle $\mathrm{G}$ as the destination vehicle that will broadcast a message received from vehicle A. As shown in Figure 2, only two relay vehicles are needed to ensure a multi-hop path between vehicle $\mathrm{A}$ and $\mathrm{G}$ (one relay node in the area $x_{2}$ and another one in the area $x_{3}$ ).

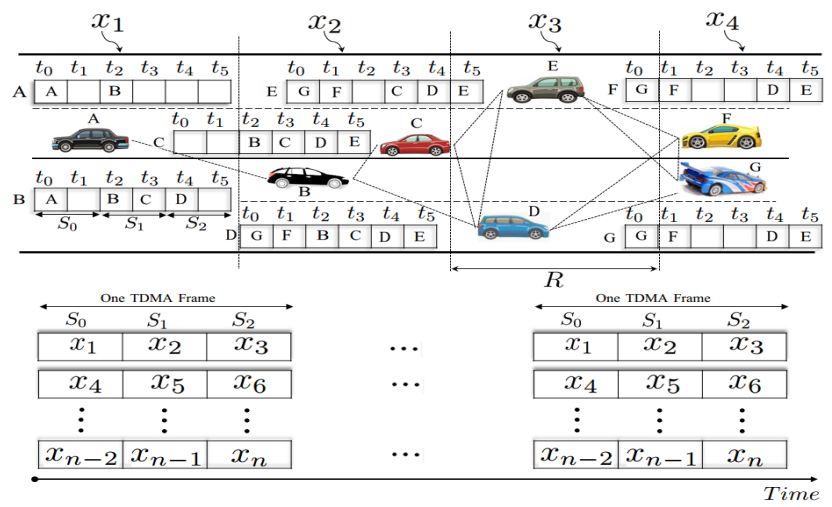

Fig. 2. VANET network using DTMAC scheduling scheme.

In the following, we suppose that the DTMAC protocol has been used by the vehicles to organize the channel access. The TDMA slot scheduling obtained by DTMAC is illustrated in Figure 2. Firstly, vehicle A forwards a packet to $\mathrm{B}$, as vehicle $\mathrm{A}$ uses its frame information to choose a vehicle that is accessing the channel during the set $S_{1}$. Upon receiving the packet for forwarding, vehicle $\mathrm{B}$ will choose by using its frame information a vehicle that's accessing the channel during the set of time slots $S_{2}$ (say vehicle D). Then, vehicle $D$ will forward the packet to $G$, as $G$ is moving in area $x_{4}$ (accessing the channel during the set $S_{0}$ ) and it is the direct neighbor of vehicle D. By using DTMAC as the MAC layer, we can note that the path A-B-D-G is the shortest, in terms of the number of hops as well as the end-to-end delay which is equal to 6 time slots (2 time slots between $t_{0}$ and $t_{2}$ as $t_{2}$ is the transmission slot for vehicle $\mathrm{B}$, then 2 time slots between $t_{2}$ and $t_{4}$ as $t_{4}$ is the transmission slot for vehicle $\mathrm{D}$ and finally 2 time slots between $t_{4}$ and $t_{0}$ as $t_{0}$ is the transmission slot in which vehicle $\mathrm{G}$ will broadcast the message received from vehicle $\mathrm{A}$ ).

The idea of TRPM is the following. Whenever a vehicle $i$ accessing the channel during the set $S_{k}$ wants to send/forward a message, it constructs two sets of candidate forwarders based on its scheduling table as follows, where $T S(j)$ indicates the time slot reserved by vehicle $j$.

- $A_{i}=\left\{j \in N(i) \mid T S(j) \in S_{(k+1) \% 3}\right\}$ // The set of vehicles that are moving in the adjacent right-hand area.

- $\quad B_{i}=\left\{j \in N(i) \mid T S(j) \in S_{(k+2) \% 3}\right\} / /$ The set of vehicles that are moving in the adjacent left-hand area.
Each source vehicle uses the position of a packet's destination and the TDMA scheduling information to make packet forwarding decisions. In fact, when a source vehicle $i$ is moving behind the destination vehicle, it will select a next hop relay that belongs to set $B_{i}$; when the transmitter is moving in front of the destination vehicle, it will select a forwarder vehicle from those in set $A_{i}$. Algorithm 1 outlines the behavior of our scheme during the procedure for forwarding messages. For each vehicle $i$ that will forward a message, we define the normalized weight function WHS (Weighted next-Hop Selection) which depends on the delay and the distance between each neighboring vehicle $j$. WHS is calculated as follows:

$$
W H S_{i, j}=\alpha * \frac{\Delta t_{i, j}}{\tau}-(1-\alpha) * \frac{d_{i, j}}{R}
$$

Where:

- $\tau$ is the length of the TDMA frame (in number of time slots).

- $\quad j$ is one of the neighbors of vehicle $i$, which represents the potential next hop that will relay the message received from vehicle $i$.

- $\Delta t_{i, j}$ is the gap between the sending slot of vehicle $i$ and the sending slot of vehicle $j$.

- $\quad d_{i, j}$ is the distance between the two vehicles $i$ and $j$, and $R$ is the communication range.

- $\quad \alpha$ is a weighted value in the interval $[0,1]$ that gives more weight to either distance or delay. When $\alpha$ is high, more weight is given to the delay. Otherwise, when $\alpha$ is small, more weight is given to the distance.

When a vehicle receives a message (as shown in Algorithm 1), it checks whether it is the destination of the packet (line 1), and if it is, it passes the packet to the upper layer (line 2). However, if the packet is destined for another vehicle, the receiver will check if the destination is moving in the same area (line 4), and if it is, the message will be transmitted immediately to its final destination (line 5). Otherwise, if the packet's destination is moving in another area, the receiver will calculate the next hop vehicle towards the destination (lines 7-11). If a relay node is found, the message will be forwarded (line 15), otherwise the message will be queued (line 17). Each forwarding vehicle includes its area ID in the relayed message. These steps are repeated by each relay vehicle until the packet is received by its final destination vehicle. To deliver a packet from a source to a destination, each vehicle $i$ receiving a message will use the weight function WSH to select a forwarding vehicle in the next area from those listed in the set $A_{i}$ or $B_{i}$. By subtracting the area ID contained in the received message, the vehicle $i$ can determine the appropriate set of potential relays. For instance, in the situation depicted in Figure 3, vehicle $T X$ will send a message to vehicle $R X$. Since, the vehicle $T X$ is moving ahead of vehicle $R X$, it will forward the message to vehicle $F 1$ that is moving in the area $x_{2}$ and accessing the channel during the set of time slots $S_{1}$. Vehicle $F 1$ needs to wait until its slot to forward the packet (i.e. it needs to wait for $T S(F 1)-T S(T X)$ slots). As vehicle $F 1$ has received 
the packet from vehicle $T X$ which is moving in the area $x_{1}$, vehicle $F 1$ will immediately select a forwarding vehicle from those located in the area $x_{3}$ which are accessing the channel during the set of time slots $S_{2}$. Then, assuming that vehicle $F 1$ decides to choose vehicle $F 2$ as the next hop to relay the packet, once the slot starts, the vehicle $F 1$ will retransmit the message to vehicle $F 2$ which in turn will forward the packet directly to its final destination $R X$.

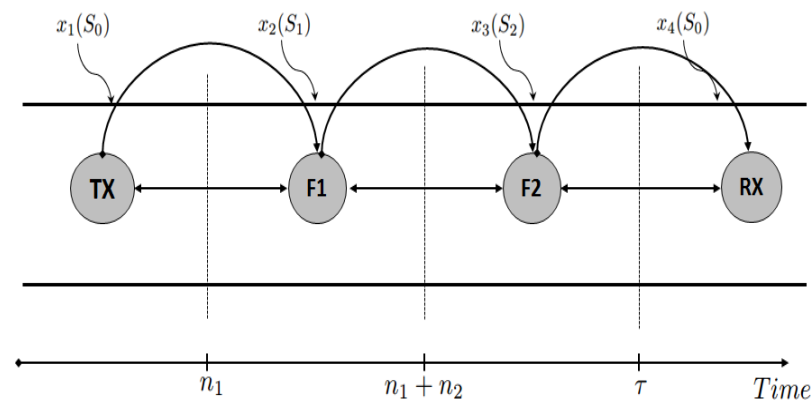

Fig. 3. Message propagation based on TDMA slot information.

$\overline{\text { Algorithm } 1 \text { Action at each vehicle which has received a }}$ safety message

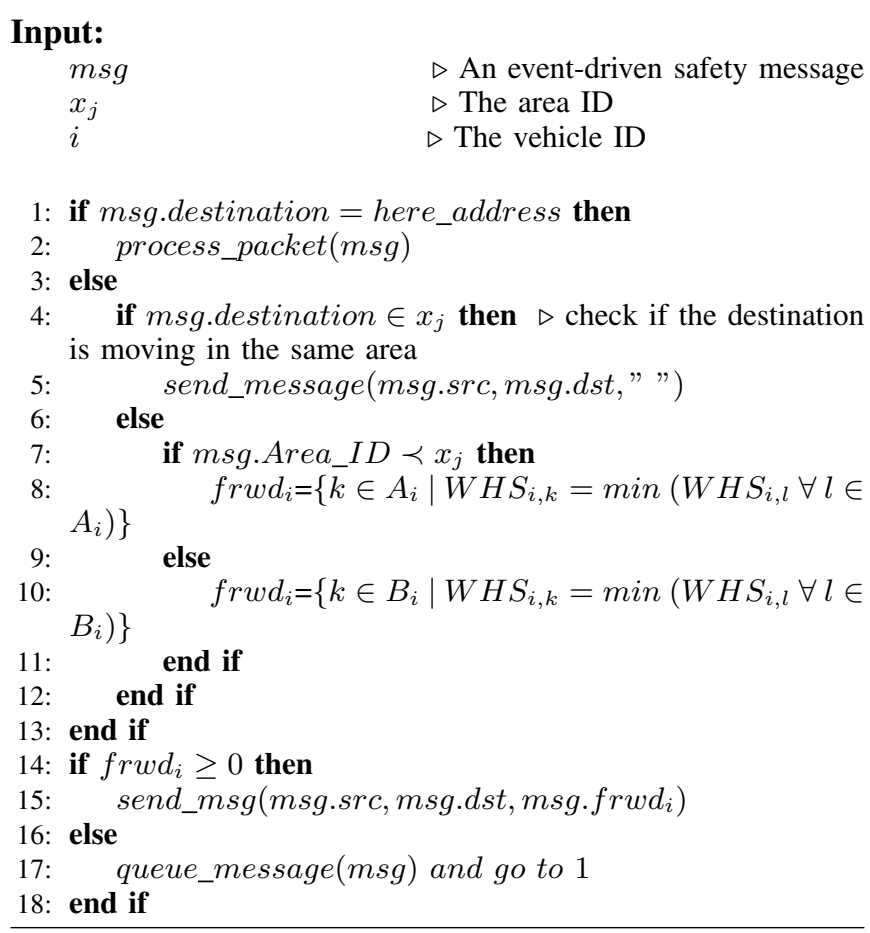

As shown in Figure 3, one frame is sufficient to deliver a message from TX to RX, because this message is forwarded three times (i.e. $n_{0}+n_{1}+n_{2}=\tau$ slots). Based on this example, we can theoretically estimate the End-to-End Delay (EED) needed to deliver a message from a source vehicle $i$ to a destination vehicle $j$. EED is estimated as follows, where $S_{d}$ is the slot duration which is fixed to $0.001 \mathrm{~s}$.

$$
E E D_{i, j} \leq\left\lceil\frac{d i s t_{i, j}}{3 * R}\right\rceil * \tau * S_{d}
$$

\section{SIMULATION RESULTS AND PERFORMANCE EVALUATION}

\section{A. Simulation Scenarios}

We generated a realistic VANET environment by selecting a real highway area from a digital map which took into account lane directions. Figure 4 shows a metropolitan area from the Map of San Jose (California) of size $3000 \mathrm{~m} \times 100 \mathrm{~m}$ exported from OpenStreetMap (OSM) and edited using Java OpenStreetMap Editor (JOSM). Then MOVE and SUMO [11] were used respectively to generate vehicular traffic scenarios and to simulate the area with vehicular traffic. Then, the traffic traces generated by SUMO were used in the $n s 2.34$ simulator. The simulation parameters used in our experiments are summarized in Table I. Each simulation run lasts for 120 seconds. After the first 2 seconds of simulation, the source vehicle starts to transmit a message 50 bytes in size. The message is transmitted to only one destination vehicle through multiple relay nodes and is repeated periodically after one second. We simulated several scenarios by varying the vehicle density between 40 and 330 vehicles in the whole network.

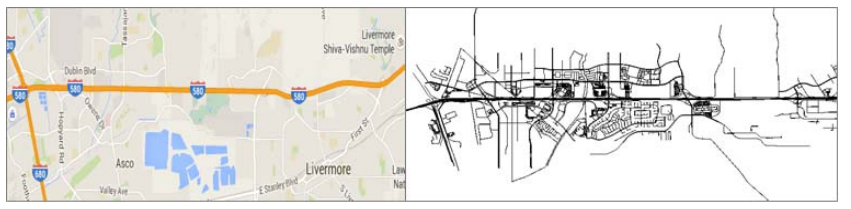

Fig. 4. San Jose (California) urban area captured from Google Maps (left) and exported to a VANET network topology by using MOVE/SUMO (right).

TABLE I. Simulation parameters

\begin{tabular}{cc}
\hline Parameter & Value \\
\hline Highway length & $3 \mathrm{~km}$ \\
Vehicle speed & $120 \mathrm{~km} / \mathrm{h}$ \\
Speed standard deviation $(\sigma)$ & $30 \mathrm{~km} / \mathrm{h}$ \\
Transmission range & $310 \mathrm{~m}$ \\
Slots/frame & 100 \\
Slot duration & $0.001 \mathrm{~s}$ \\
Simulation time & $120 \mathrm{~s}$ \\
\hline
\end{tabular}

\section{B. Performance evaluations}

We compared the proposed TRPM with two multi-hop communication protocols that have the same underlying principle (i.e. MAC-aware routing protocol). The first one is the Random TDMA aware routing protocol, called RTDMA [4]. In this protocol the time slots are allocated to vehicles randomly and the next hop decision for unicast traffic forwarding is based on the vehicles' positions and the time slot information from the random TDMA scheduling. The second protocol is the Contention aware routing protocol, called CRP [12] which is based on classical flooding, where every vehicle relays each packet received to all its one-hop neighbors at least once until the packet has been received by its final destination vehicle. These protocols are evaluated by varying the Source-to-Destination Distance (SDD) between $550 \mathrm{~m}$ and $2550 \mathrm{~m}$. Moreover, we evaluated these protocols on the same network scenarios in terms of the average EED, average number of hops and average delivery ratio. The performance of TRPM depends on the value of $\alpha$, which determines its 
behavior. In fact, when $\alpha$ is high, more weight is given to the delay. Otherwise, when $\alpha$ is small, more weight is given to the distance and thus to the number of hops. For this, we evaluated several values of $\alpha$ in different scenarios to find the optimal value of this parameter. Figure 5-a shows the variation of the average EED with the change in $\alpha$ values. We can see from this figure that the EED is reduced to less than $150 \mathrm{~ms}$ by choosing values of $\alpha$ between 0.3 and 0.6 under a high traffic condition scenario. However, when $\alpha<0.3$ or $\alpha>0.6$ the average EED is high, the reason being that when $\alpha$ is small, more weight is given to the distance, hence the selected relay vehicles between the source and the destination generate more delay due to the high gaps between their sending slots. On the other hand, higher values of $\alpha$ give more weight to the delay than to the distance which provides routes that have a good delay but a greater number of relay vehicles as we can see in Figure 5-b. In the following, we present the simulation results and we analyze the performance of our proposed protocol. For these results, the weight factor $\alpha$ was fixed to 0.4 .

Figure 6-a shows the average EED for all the multihop communication protocols under consideration. We can note from this figure that the TRPM protocol performs very well compared to RTDMA and CRP, especially as the distance between the source and destination increases. For instance, when $S D D=2295 \mathrm{~m}$, the TRPM protocol achieves an average EED of $234.16 \mathrm{~ms}$ while RTDMA and CRP show an average delay of $520.33 \mathrm{~ms}$ and $626.3 \mathrm{~ms}$, respectively (i.e. approximately $122.2 \%$ and $167.47 \%$ higher than TRPM). This is mainly because, as discussed in Section 4 , the proposed protocol can reduce the gap between the sending slots of relay vehicles that are moving in adjacent areas by dividing the frame into three sets of time slots. Moreover, we can observe that RTDMA performs much better than CRP. These results can be explained by the fact that, in CRP, all candidate relay nodes are considered without taking into account any criteria. This figure also compares the theoretical values of average EED with those obtained by simulation. The theoretical values are close to the simulated values for all shown SDDs. In Figure 6-b, we show the relationship between the average number of relay nodes and the SDD. It is clear from this figure that the number of relay vehicles increases as the distance increases. We can note that TRPM can significantly reduce the number of relay vehicles required to deliver a message compared to RTDMA and CRP protocols. This is due to the fact that TRPM always selects only one relay vehicle for each area (i.e. one relay node for each $310 \mathrm{~m}$ ), in contrast to the RTDMA and CRP in which two or more relay vehicles can be successively selected within the same area. Unlike CRP and RTDMA, these two figures clearly show that TRPM achieves better performances in terms of both average EED and average number of hops, since it uses a next-hop selection function that can balance the two metrics studied.

In order to assess the effect of collision in the performance of these protocols, we evaluate then on the following scenario where there is a background traffic that consists of a periodic message broadcasted by each vehicle every $100 \mathrm{~ms}$. Figure 7 shows the average number of relay vehicles for TRPM, RTDMA and CRP protocols when varying vehicle density. Unlike RTDMA and CRP, the average number of hops in TRPM is still constant as vehicle density increases.
This is mainly due to the forwarding concept in TRPM which always selects only one forwarding vehicle in each area so that the number of relay vehicles always remains constant.

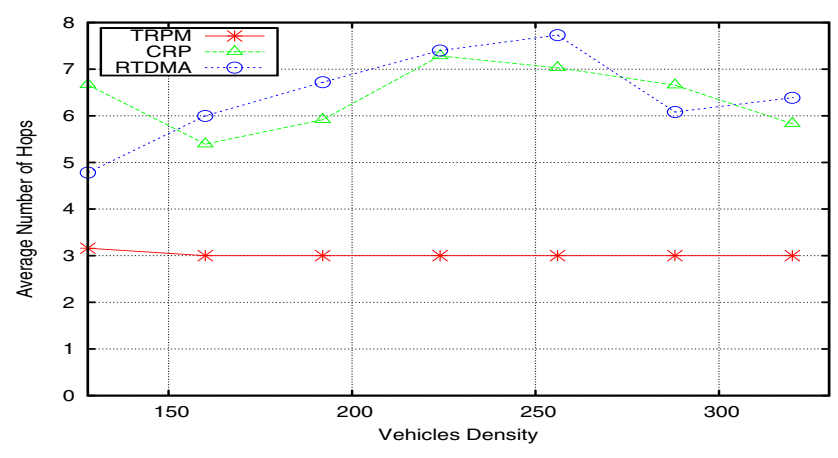

Fig. 7. The average number of relay vehicles vs vehicle density.

In order to validate the previous results, we evaluate the performance of these protocols in terms of delivery reliability. Figure 8 shows the average delivery ratio of the three protocols under consideration when varying vehicle density. As shown in this figure, TRPM achieves a considerably higher delivery rate of packets than RTDMA and CPR. For instance for a high density (in the case of 280 vehicles), the TRPM protocol achieves an average delivery ratio of $98.4 \%$, in contrast to RTDMA and CRP which show a rate of $75.91 \%$ and $65.52 \%$, respectively. We can note that TRPM maintains almost an average delivery ratio close to the ideal rate (i.e. $100 \%$ ) for all VANET scenarios. This is because TRPM implements an optimized relay vehicle selection mechanism that can avoid redundant transmissions. Moreover, TRPM is a contention-free based protocol that can reduce packet collisions in the presence of background traffic. We can also see that the CRP and RTDMA protocols have very poor performances. These results might well be expected for CRP since it is a flooding based routing protocol in which each vehicle retransmits the message received to all its neighboring vehicles without using any selection mechanism.

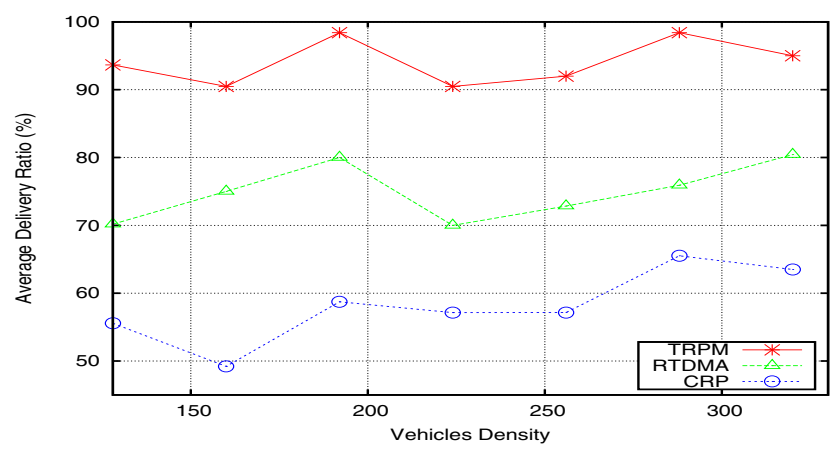

Fig. 8. The average delivery ratio vs vehicle density.

\section{CONCLUSION}

The stringent requirements of VANET safety applications mean that their messages need to be delivered quickly and with a high degree of reliability. However, designing an efficient multi-hop communication protocol for packet delivery is a major challenge in VANETs due to the rapid changes 


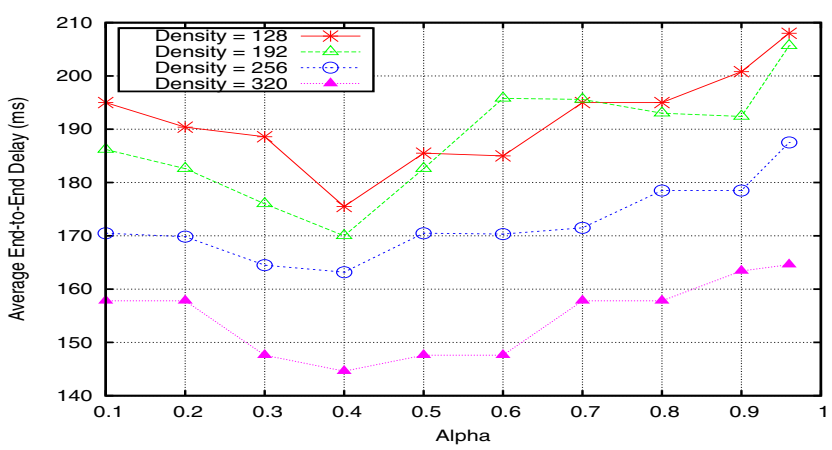

(a)

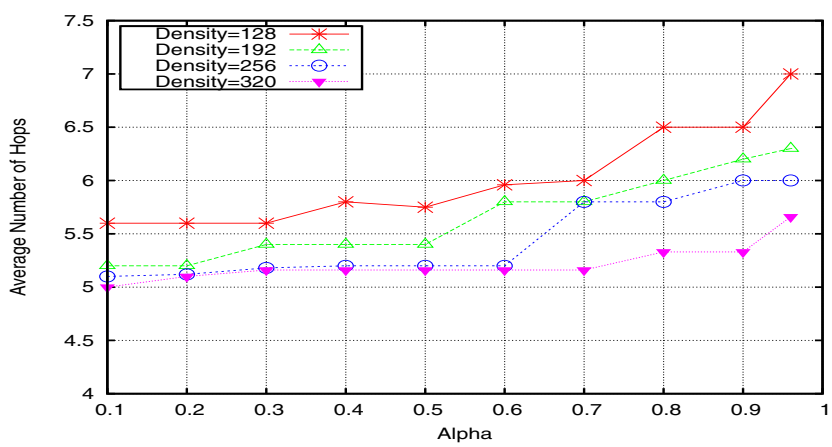

(b)

Fig. 5. Effect of changing $\alpha$ on average EED (left) and on average number of hops (right).

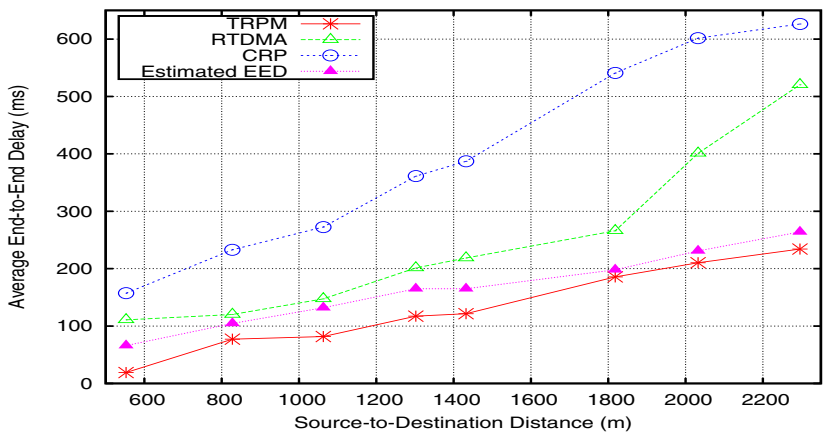

(a)

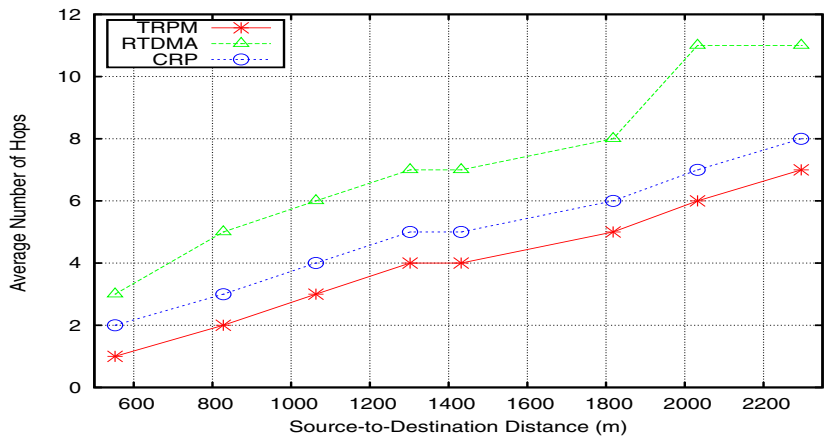

(b)

Fig. 6. The average EED (left) and the average number of relay vehicles (right) vs SDD.

in network topology and the lack of infrastructure. In this paper, we propose a novel TDMA aware routing protocol to allow a vehicle to send a packet over a long distance through multiple relay vehicles. The packet is delivered from a source vehicle to a destination vehicle using the geographic positions and the time slot information from the TDMA scheduling. Moreover, our protocol takes into account the relay selection efficiency by using a weighted next-hop selection function in order to make coherent next hop decision in terms of both number of relay vehicles and end-to-end delay (EED). The simulation results show that, compared to two other protocols, our proposed cross layer protocol provides better performances in terms of average end-to-end delay, average number of hops and average delivery ratio.

In future work, we will explore how our TDMA aware routing protocol can be adapted to support multichannel operation and to provide a reliable delivery of messages on both the control and service channels. Moreover, we plan to carry out extensive simulations to compare it with a geographic routing protocol, such as the Greedy Perimeter Stateless Routing (GPSR) protocol.

\section{REFERENCES}

[1] M. Hadded, P. Muhlethaler, A. Laouiti, R. Zagrouba, and L. A. Saidane, "TDMA-based MAC protocols for vehicular ad hoc networks a survey, qualitative analysis and open research issues," IEEE Communications Surveys Tutorials, vol. 17, no. 4, pp. 2461-2492, Jun. 2015.

[2] M. Hadded, A. Laouiti, P. Muhlethaler, and L. A. Saidane, "An infrastructure-free slot assignment algorithm for reliable broadcast of periodic messages in vehicular ad hoc networks," in Vehicular Technology Conference VTC-Fall, Montreal, Canada, Sep. 2016.
[3] L. Laouil, V. Felea, J. Bernard, and H. Guyennet, "Mac-aware routing in wireless sensor networks," in BlackSeaCom, Constanta, Romania, May 2015, pp. 225-229.

[4] I. Rhee, A. Warrier, J. Min, and L. Xu, "DRAND: Distributed randomized tdma scheduling for wireless ad hoc networks," in ACM MobiHo, New York, May 2006, pp. 190-201.

[5] 802.11p-2010, IEEE standard for information technology - Telecommunications and information exchange between systems - local and metropolitan area networks - specific requirements part 11 : Wireless LAN medium access control (MAC) and physical layer (PHY) and physical layer (PHY) specifications amendment 6 Std., 2010.

[6] R. Uzcátegui and G. Acosta-Marum, "WAVE: A tutorial," IEEE Communications Magazine, vol. 47, no. 5, pp. 126-133, May 2009.

[7] B. Blaszczyszyn, A. Laouiti, P. Muhlethaler, and Y. Toor, "Opportunistic broadcast in VANETs (OB-VAN) using active signaling for relays selection," IEEE International Conference on ITS Telecommunications (ITST08), pp. 384-389, Oct. 2008.

[8] H. A. Omar, W. Zhuang, and L. Lio, "On multihop communications for in-vehicle internet access based on a TDMA MAC protocol," in IEEE Conference on Computer Communication (INFOCOM), Toranto, Canada, Apr. 2014, pp. 1770-1778.

[9] W. Zhuang, H. A. Omar, and L. Lio, "VeMAC: A novel multichannel MAC protocol for vehicular ad hoc networks," in IEEE Conference on Computer Communications Workshops (INFOCOM WKSHPS), Shanghai, China, Aug. 2011, p. 413418.

[10] B. Karp and H. Kung, "GPSR: greedy perimeter stateless routing for wireless networks," in international conferences on Mobile Computing and Networking (MobiCom), Boston, Massachusetts, USA, 2000, pp. 243-254.

[11] F. Karnadi, Z. Mo, and K. chan Lan, "Rapid generation of realistic mobility models for VANET," in IEEE WCNC, Hong Kong, China, Mar. 2007, pp. 2506-2511.

[12] Y. B. Ko and N. H. Vaidya, "Flooding-based geocasting protocols for mobile ad hoc networks," Mobile Networks and Applications, vol. 7. 